\title{
I miss you babe: Analyzing Emotion Dynamics During COVID-19 Pandemic
}

\author{
Lynnette Hui Xian Ng ${ }^{1} \quad$ Roy Ka-Wei Lee $\quad$ Md Rabiul Awal $^{3}$ \\ ${ }^{1}$ Carnegie Mellon University \\ ${ }^{2}$ Singapore University of Technology and Design \\ ${ }^{3}$ University of Saskatchewan \\ lynnetteng@cmu.edu, roy_leedsutd.edu.sg, mda219@usask.ca
}

\begin{abstract}
With the world on a lockdown due to the COVID-19 pandemic, this paper studies emotions expressed on Twitter. Using a combined strategy of time series analysis of emotions augmented by tweet topics, this study provides an insight into emotion transitions during the pandemic. After tweets are annotated with dominant emotions and topics, a timeseries emotion analysis is used to identify disgust and anger as the most commonly identified emotions. Through longitudinal analysis of each user, we construct emotion transition graphs, observing key transitions between disgust and anger, and self-transitions within anger and disgust emotional states. Observing user patterns through clustering of user longitudinal analyses reveals emotional transitions fall into four main clusters: (1) erratic motion over short period of time, (2) disgust $\rightarrow$ anger, (3) optimism $\rightarrow$ joy. (4) erratic motion over a prolonged period. Finally, we propose a method for predicting users subsequent topic, and by consequence their emotions, through constructing an Emotion Topic Hidden Markov Model, augmenting emotion transition states with topic information. Results suggests that the predictions fare better than baselines, spurring directions of predicting emotional states based on Twitter posts.
\end{abstract}

\section{Introduction}

In August 2020, the COVID-19 pandemic raged throughout the world. Caused by the virus SARSCoV-2 (Mehta et al., 2020), the pandemic, still ongoing during this study, has infected more than 26 million people and has taken over 800,000 lives. Globally, countries have taken unprecedented measures for virus containment, most notably, implementing cities lockdown and forcing citizens into isolation.

Despite limited face interactions, digitalization has provided a space for expression. Twitter users have expressed their yearning for loved ones, their lifestyles, the freedom of social activities and travels: "[...] I miss you babe. I wish this shit is over and we will travel Himalay together!. Others express frustration about missed opportunities and the stress of isolation: \#covid [..] will kill but \#starvation WILL definitely kill.

Previous studies of emotion analysis on Twitter largely focused on identifying emotions for general tweets (Sailunaz et al., 2018; Colneric and Demsar, 2018; Subramaniam et al., 2017), and during the COVID-19 period (Mukherjee et al., 2020; Gupta et al., 2020). In analyzing emotion dynamics on Twitter, Naskar et al. (2019) modelled emotional states of Twitter users with a Hidden Markov Model, and further showed that Twitter users change their emotional state against their topics (Naskar et al., 2020).

Our study aims to characterize emotional states and transitions of Twitter users during the global COVID-19 lockdown. The study contributes to ongoing literature through the following research questions: (1) What are the emotion dynamics expressed amid the COVID-19 pandemic? That is, what are the topics discussed and the corresponding emotions? (2) What are the profiles of emotion transitions over time? (3) How might we effectively predict a user's subsequent tweet's emotion?

To investigate the above questions, we collected a Twitter dataset during the COVID-19 pandemic (Section 3) to study emotion dynamics, which is the interplay between topics discussed and emotions expressed in a tweet. We computationally annotated our collected tweets with emotions from an emotion classifier pre-trained on an labeled emotion dataset (Mohammad et al., 2018). To augment the emotion label, we learned topics of tweets using Latent Dirichlet Allocation (Mimno and Mimno, 2013). In Section 4, we study user emotion transitions by first constructing an aggregate emotion 
transition graph across all users, representing emotions as nodes and edges as emotion transitions between user tweets. We then cluster users' emotion transition graphs and observe emotional transition patterns. In Section 5, we propose an Emotion Topic Hidden Markov Model (ET-HMM), adopting a Hidden Markov Model (Rabiner, 1989) on the users' emotion transition states augmented with topic information, and evaluate the model's prediction of the topic and emotion expressed in a user's subsequent tweet. Our findings, as discussed in Section 6, suggest that the ET-HMM outperform baselines, and are hopefully useful in monitoring emotional health through social media.

\section{Related Work}

Emotion analysis in text is a widely studied area, spanning the evolution of emotions, emotion models, emotion detection methods and construction of datasets, detailed in several surveys highlighting the methods and challenges (Sailunaz et al., 2018; Binali et al., 2010; Kao et al., 2009; Jain et al., 2017).

Twitter, a text-rich social media, is a valuable and popular data source for emotion analysis (Sailunaz and Alhajj, 2019; Colneric and Demsar, 2018; Subramaniam et al., 2017). Previous studies have proposed methods to detect and analyze Twitter users' emotional responses towards an event (Jones et al., 2016) and understand users' mental health (Wang et al., 2016; Seabrook et al., 2018). Colnerî̀ and Demsar (2018) investigated and compared classification methods to recognize eight expressed emotions: joy, sadness, trust, disgust, fear, anger, surprise, anticipation.

Besides classifying user emotions on Twitter, existing studies analyzed emotion dynamics and changes of user emotions over time. Naskar et al. (2020) investigated social dynamics of emotions in Twitter users' opinions, and attempted to change user emotions towards social issues over time. During the pandemic, Mukherjee et al. (2020) analysed emotion trends on Indian tweets and the influence of public emotions on individual emotions, while Gupta et al. (2020) performed emotion and topical analysis on over 63 million tweets. Naskar et al. (2019) proposed a Hidden Markov Model (HMM) to model emotional changes through a Twitter user's consecutive sequence of tweets.

This study contributes to ongoing emotion analysis research by characterizing Twitter users' emo- tion dynamics during the COVID-19 pandemic. More specifically, we provide a preliminary understanding of the transition of emotional and mental states as the global pandemic develops. To the best of our knowledge, this is the first study that examines emotional dynamics amid a pandemic event.

\section{Data Collection}

We adopt the Twitter dataset collected in (Awal et al., 2020). Specifically, we begin with a random sample of 5.4 million tweets from 10.1 million users taken from the $1 \%$ Twitter streaming API collected during a period of seven weeks beginning from March 22, 2020. The tweets were collected with the following hashtags: '\#covid19', '\#COVID-19', '\#COVID', '\#corona', '\#Coronavirus', '\#coronavirus', '\#CoronaVirus'. From these tweets, we selected for tweets that contained the English words related to "yearning" and their related word forms (i.e. miss, yearn, pine, long, wish, crave), so as to ensure that a substantial number of tweets express some emotions (i.e. do not classify as neutral), and a spectrum of emotions is expressed. In this first selection of data, we obtained 2.8 million tweets from 233,932 users.

Using the selected set of users, we extracted all their tweets during our collection period, to facilitate longitudinal user emotion dynamic studies. This process yielded us a total of 3,035,844 tweets from 233,932 unique users. We thus use this consolidated dataset for further analysis and experiments.

\section{Characterizing Emotion Dynamics}

\subsection{Topic Discovery}

To discover topics in the datasets, we apply the Mallet LDA topic modelling tool (Mimno and Mimno, 2013) to learn topic clusters and their corresponding keywords. Each topic is represented by a probability distribution over the entire vocabulary, and created a topic model with the number of topics derived through the elbow rule from model coherence scores.

In the initial selected set of tweets, the topic analysis revealed four dominant topics, signifying the entities Twitter users yearn for. These topic clusters are: (1) hope: hope, find, story; (2) transition to online: online, call, show, work; (3) life and death: life, die, religion, helpless, crisis; (4) activities and people missed: summer, friend, family, future. 
Table 1: Distributions and sample keywords of topics

\begin{tabular}{|l|l|l|l|}
\hline & Topic & Keywords & $\begin{array}{l}\text { Tweets } \\
\text { (\%) }\end{array}$ \\
\hline 1 & General & $\begin{array}{l}\text { medium, news, social, response, government, order, fact, } \\
\text { question }\end{array}$ & 9 \\
\hline 2 & Vaccines and Testing & testing, human, vaccine, risk, treatment, drug, infection & 9 \\
\hline 3 & Country specific & country, world, safe, good, home, spread, nation, end & 10 \\
\hline 4 & Lockdown & lockdown, government, police, citizen, fight, regime, law & 10 \\
\hline 5 & Call for leader & people, time, crisis, work, great, leader, support, action & 9 \\
\hline 6 & High case numbers & death, case, positive, total, high, bad, rate & 10 \\
\hline 7 & Elections & trump, call, election, free, mask, pandemic & 10 \\
\hline 8 & Relationships & people, life, year, family, friend, good & 12 \\
\hline 9 & $\begin{array}{l}\text { Concern for healthcare } \\
\text { workers }\end{array}$ & $\begin{array}{l}\text { patient, doctor, hospital, health, care, worker, long, hour, } \\
\text { medical, staff }\end{array}$ & 11 \\
\hline 10 & $\begin{array}{l}\text { Business and job re- } \\
\text { liefs }\end{array}$ & $\begin{array}{l}\text { state, economy, business, job, global, pandemic, money, } \\
\text { relief }\end{array}$ & 12 \\
\hline
\end{tabular}

As the tweets are short, we assume each tweet only contain one topic and we annotate the tweet with the most dominant topic, i.e., the topic with the highest percentage weightage. This topic information per tweet is used to augment the Emotion Topic Hidden Markov Model, drawing on the fact that tweet topics can significantly influence expressed emotions (Naskar et al., 2020). The final dataset yielded a topic cluster of 10 topics, presented in Table 1.

\subsection{Emotion Profiling}

Emotion Annotation. To perform emotion profiling, we first annotate all tweets with their emotions. We constructed an Emotion Annotation Model based on SemEval-2018 (Mohammad et al., 2018), which contains English tweets that have been manually annotated with 11 emotions: anger, anticipation, disgust, fear, joy, love, optimism, pessimism, sadness, surprise, trust and neutral.

For the Emotion Annotation Model, we express each tweet as a vector representation of GloVe embeddings (Pennington et al., 2014). Then we built a two-fold deep-learning classifier using Keras Tensorflow on the training dataset. The first fold consists of a 100-unit Bidirectional Long-Short-TermMemory (LSTM) followed by a 200-filter unit Convolutional Neural Network (CNN). The second fold consisted of a 100-filter unit CNN combined with a 100-unit Bidirectional LSTM. The two folds are combined with an Attention layer, and a consistent 0.3 dropout rate is used in both folds. With this model, we obtained an accuracy of $84 \%$ on the test dataset provided. Each tweet is annotated with the corresponding emotions, and its dominant emotion is determined as the emotion class with the highest percentage weightage. The tweets that are deemed not to display any emotions are annotated as neutral. While the original annotations had 11 categories, our final emotion annotation contains only seven emotion categories as the rest do not surface as dominant emotions in the tweets. Figure 1 shows the distribution of tweets and its annotated emotions. We note that the tweets are mostly dominated by anger, which is a reasonable emotion during this trying times. We further note that fear is present but in very low proportions.

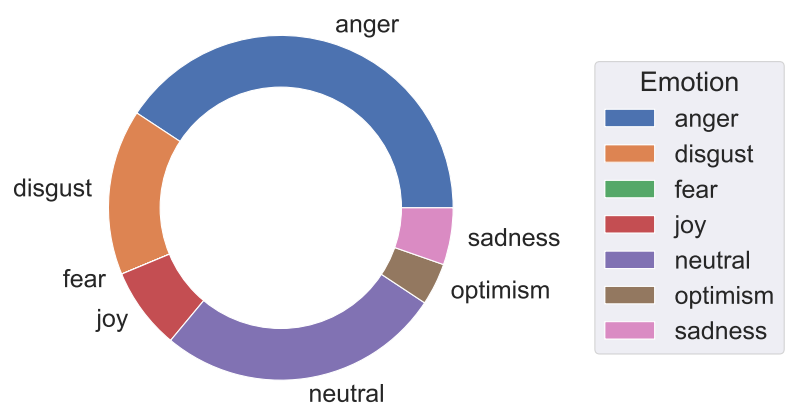

Figure 1: Distribution of tweets by emotions

Singular Emotion Dynamics. Emotion dynamics corresponding to a singular emotion is represented with the topic distribution across the seven emotional categories (Figure 2). Tweets expressing anger and disgust are discuss the spectrum of all 10 topics. Tweets exhibiting fear have a high pro- 


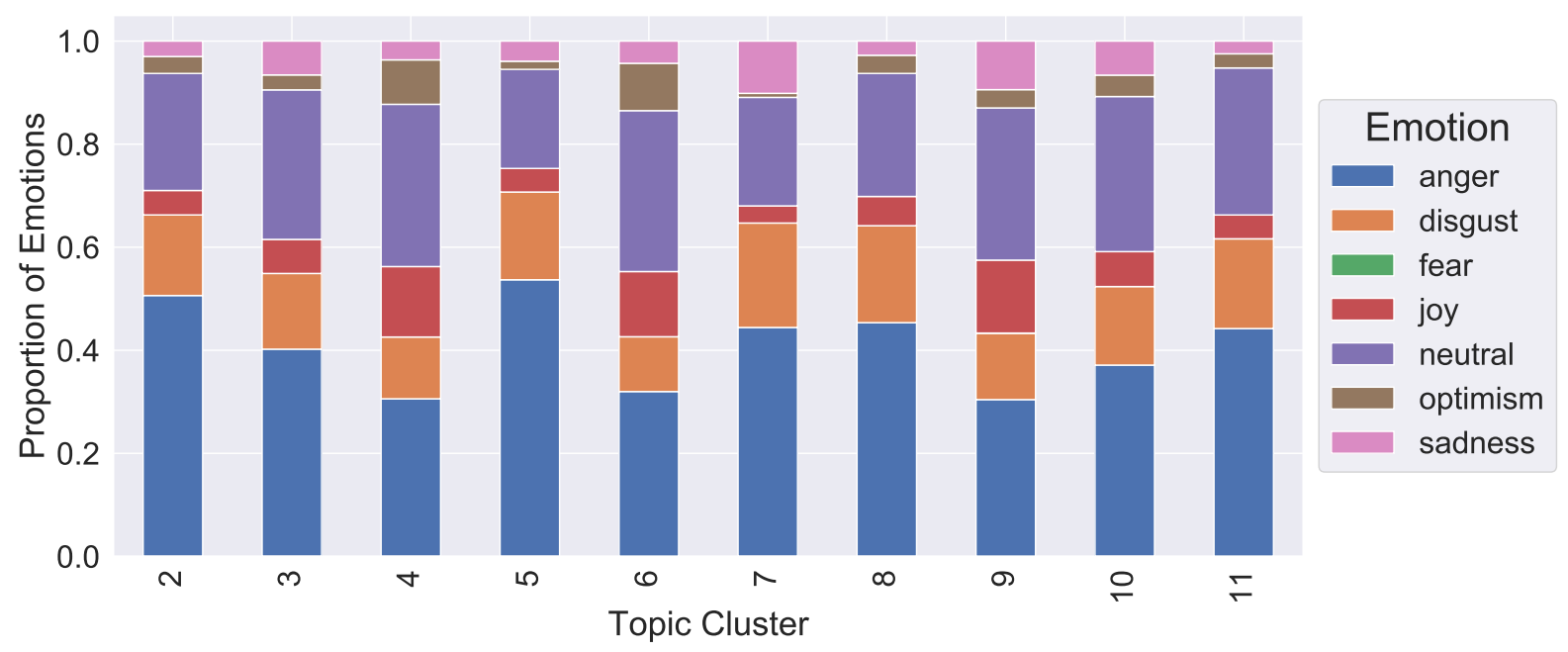

Figure 2: Emotion distribution across topics.

portion of discussion around topics 9 and 10 (concern for healthcare workers; business/ job reliefs). Anger is observed to be the dominating sentiment towards the lockdown (topic 4) and business/ job reliefs (topc 10). Relationship discussions dominate sad and joyful tweets, situating users emotional states when talking about loved ones.

Emotion Transitions across the Corpus. We begin our profiling of an aggregate of emotion transitions during the month of March by first constructing a longitudinal study of emotions per user. For each user, we construct an emotion transition graphs using a chronological trail of his collected tweets, representing emotions as nodes and emotion transitions between tweets as links. We ignore users that have a trail length of two or less, where the emotion trail is too short. The transition probability from emotion $e_{1}$ to the emotion $e_{2}$ indicates the probability that a Twitter user writes a tweet at time $t$ of emotion $e_{1}$ and a tweet at the next time step, $t+1$, with emotion $e_{2}$. To reduce graph complexity, we generated an undirected graph, that is, we aggregated transitions of disgust $\rightarrow$ anger and anger $\rightarrow$ disgust together. The summation of individual user graphs produces Figure 3, an aggregated emotion transition graph across the corpus.

In this corpus, the emotions that are expressed most frequently are: anger comprising of $33.3 \%$ (1.01 million) tweets, and disgust with $34.4 \%$ (1.04 million) tweets. The least frequent observed emotion is fear with only 11 tweets. Joy, optimism and sadness are observed around $15.3 \%$ of the time. During our analysis timeframe, the disgust-anger transition is most prominent with 913,867 tweets, and the optimism-fear transition is the least frequent with 121 counts.

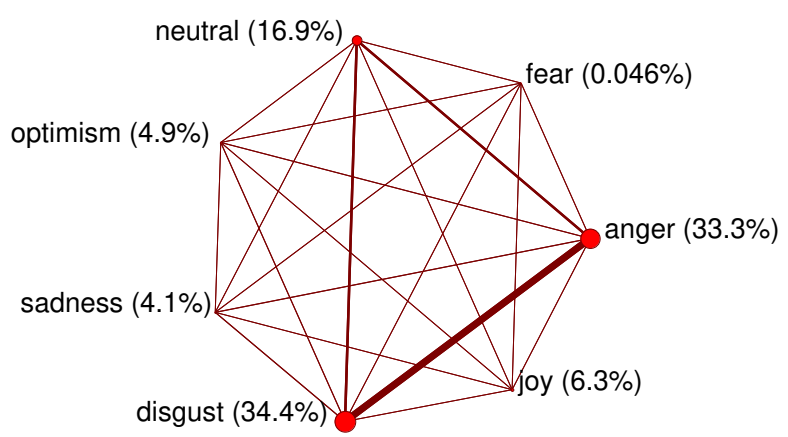

Figure 3: Aggregated Emotion Transition Graph. Width of links represent the frequency of transition, size of node show the number of tweets annotated with the emotions.

Patterns of "Mood Swings". We characterize profiles of user emotion transitions over time, observing patterns of "mood swings". Representing each user's sequence of emotion as a trail, we clustered all the trails together using a trail depth of 1 and a $60 \%$ similarity in the trail (Gullapalli and Carley, 2013), using the software ORA (Carley, 2014). A trail depth of 1 compares each emotion singularly and the similarity thresholds the length of trail overlap to be clustered together. With these parameters, the trail (disgust $\rightarrow$ anger $\rightarrow$ disgust $\rightarrow$ optimism $\rightarrow$ joy) is clustered together with (disgust $\rightarrow$ anger $\rightarrow$ disgust $\rightarrow$ anger $\rightarrow$ disgust) because $60 \%$, or 3 out of 5 , of the emotion transitions overlap.

Figure 4 displays the "mood swing" profiles in a chronological Emotion Clustering Chart. In this time-series representation, four main clusters are 
observed: (1) erratic emotion transitions across several emotion states over a short period of time; (2) emotion transitions between disgust and anger; (3) emotion transitions between optimism and joy; (4) erratic emotion transitions across several emotion states across a prolonged period of time. Table 2 shows example tweets from three representative users from these clusters.

Longitudinal Emotion Dynamics. We reflect emotion dynamics corresponding to this longitudinal study of emotion transitions as dominant topics for each emotion transition (Table 3). Clusters 2 and 4 exhibit different profiles, where the former alternates between two emotions while the latter displays "mood swings", yet the topics discussed are the same. Where users exhibit "mood swings" over a short time period, the topics are very focused on the medical science of the virus, and users alternating between optimism and joy exudes hope for people (doctors, healthcare workers, government).

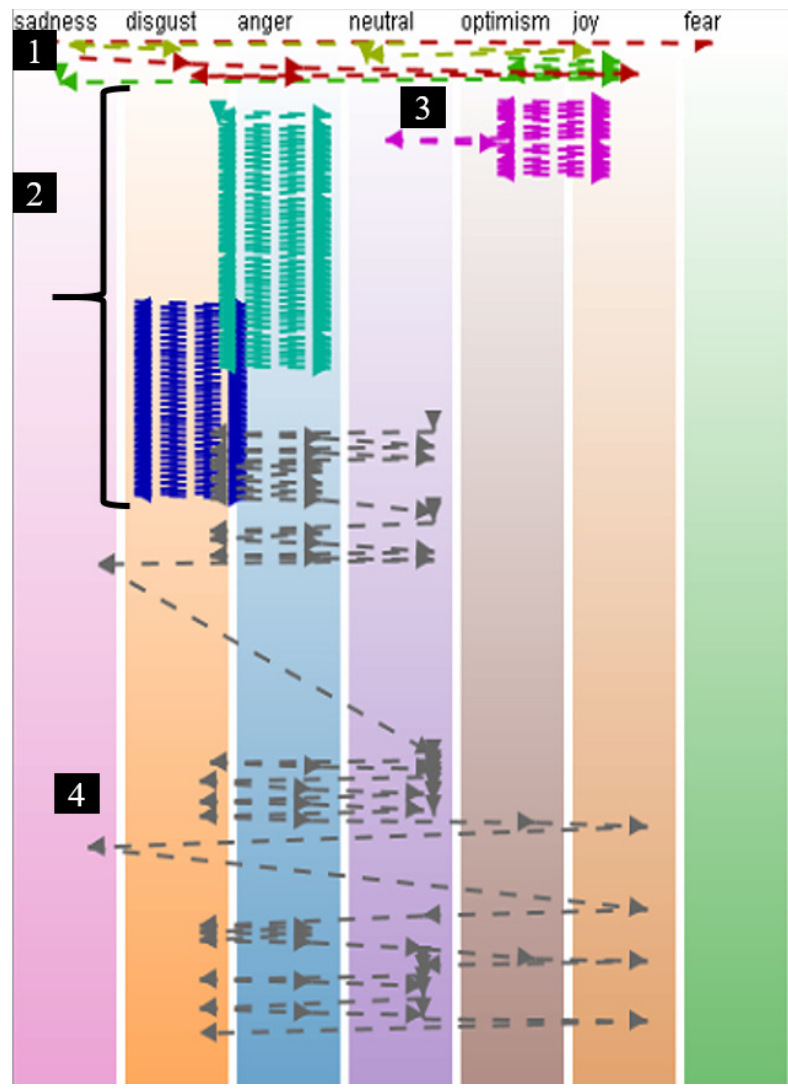

Figure 4: Emotion Clustering Chart in chronological order. Each trail is a group of users that exhibit similar longitudinal emotional profiles.

\section{Predicting Emotion Dynamics}

We describe the method and results in predicting emotion dynamics through a proposed Emotion
Topic Hidden Markov Model (ET-HMM) framework. We evaluate our ET-HMM against Naive Bayesian and Support Vector Classifier models.

Emotion Topic Hidden Markov Model. We model user emotion state transitions using a probabilistic sequence model: the Markov Model (Rabiner, 1989). In the Markov model, the exhibition of the next emotional state is based on the current state, defined by the transition probabilities of moving from one state to another across neighbouring time periods. We augment the user transition markov model with discrete distributions of dominant topics as hidden states, constructing an Emotion Topic Hidden Markov Model (ET-HMM) ${ }^{1}$, which reflects the importance of topics on emotions, to explore user feelings when they tweet on a particular topic.

Figure 5 shows the result of the ET-HMM constructed using the Pomegranate python library (Schreiber, 2018). We observe that the emotions anger and neutral has the highest initial probability, meaning when a random user first tweets, he is likely to post a tweet with these emotions during the pandemic. With the exception of fear, all emotions are equally as likely to be found at the last tweet of a trail. If a user is in an angry state, the probability of staying in the same state in subsequent tweets is higher than other emotional states.

Emotion Prediction with ET-HMM. We perform a predictive task using our ET-HMM model. Given a user's past emotion dynamics trail consisting of a sequence of topics and emotions, we attempt to predict his subsequent emotion dynamic. We construct an emotion dynamic trail for each user using the dominant topic and emotions for each tweet. We then remove the final tweet's emotion dynamics from the trail, to be used as the test data point for the sequence. Using the Viterbi algorithm, we predict the emotion dynamic of each user, given his emotion dynamic trail. We select the states that have the maximum probability of occurring given the trail of previous topics and emotions.

Baseline Emotion Prediction. We evaluate the performance of our ET-HMM framework by comparing against a multinomial Naive Bayesian (NB) classifier and a Support Vector Classifier (SVC). We constructed a 7 emotion $\times 10$ topics $=70$ dimension matrix, and filled its rows with the (topic, emotion) of each user at the particular time period.

\footnotetext{
${ }^{1}$ Code: https://gitlab.com/bottle hop/wellness/ethmm
} 
Table 2: Emotion transition clusters with illustrations from three users.

\begin{tabular}{|l|l|}
\hline Tweet text & Emotion \\
\hline \hline Example Tweets from user_I (Cluster 2: disgust $\rightarrow$ anger) & \\
\hline Mental health issues raging in India among \#COVIDIOTS Before and after \#COVID-19 & Disgust \\
\hline He wants to starve Indians to die before \#COVID-19 can kill them & Anger \\
\hline $\begin{array}{l}\text { Till 31st March - yes Stop ferrying back Indians from different countries to destroy our } \\
\text { Stage } 3 \text { and make it Stage 1 again }\end{array}$ & Disgust \\
\hline $\begin{array}{l}\text { \#covid MAY strike and Without testing and treatment, will kill but \#starvation WILL } \\
\text { definitely kill }\end{array}$ & Anger \\
\hline \hline Example Tweets from user 2 (Cluster 3: optimism $\rightarrow$ joy) & \\
\hline $\begin{array}{l}\text { Thankful to be able to help in any possible way during these tough times. - Use Wysa to } \\
\text { vent or just talk through negative thoughts. Let it help you cope with pandemic anxiety } \\
\text { and lockdowns. It is anonymous, safe and free. \#covid \#mentalhealth }\end{array}$ & Joy \\
\hline $\begin{array}{l}\text { We know that uncertainty can be worrying but let's try to be patient and be hopeful, } \\
\text { brighter days could be just around the corner! RT and share this with someone who needs } \\
\text { to hear this! \#corona \#Covid-19 \#hope \#uncertainty \#positivity }\end{array}$ & Optimism \\
\hline $\begin{array}{l}\text { Every cloud has a silver lining, doesn't it? Let's focus on the positive and stay hopeful! } \\
\text { RT and share it with someone who needs to see this! \#silverlinings \#covid \#hope } \\
\text { \#positivity }\end{array}$ & Optimism \\
\hline \hline Example Tweets from user 3 (Cluster 1, 4: erratic motions) & \\
\hline $\begin{array}{l}\text { @madus661 @ LizClaman MyPOV: correct. But aren't there provisions in many privacy } \\
\text { policies and legislation that enables anonymized aggregate data sharing without explicit } \\
\text { opt in consent. \#privacy vs \#publichealth \#coronavirus \#covid19 }\end{array}$ & Disgust \\
\hline $\begin{array}{l}\text { MyPOV; Tech putting its might in the war on \#coronavirus @ IBM helps bring \#super- } \\
\text { computers into the global fight against COVID-19 \#covid19 }\end{array}$ & Anger \\
\hline MyPOV: rediscovering that piano. \#shelterinplace \#weekend \#coronavirus \#covid19 & Joy \\
\hline $\begin{array}{l}\text { MyPOV: a kale salad, more leftover fried rice \#ShelterInPlace \#coronapocalypse \#covid19 } \\
\text { \#coronavirus }\end{array}$ & Neutral \\
\hline $\begin{array}{l}\text { [... as for response get set of the \#postpandemic playbook. We will reveal more next } \\
\text { week. Have been meeting with CEOs }\end{array}$ & Optimism \\
\hline
\end{tabular}

For both the NB and SVC, we used the matrix constructed at time $t_{n-3}$ and $t_{n-2}$ as a training set, representing the (topic, emotion) transition from time $t_{n-3}$ and $t_{n-2}$, and performed predictions on the matrix at time step $t_{n-1}$. The predicted matrix is compared with the actual matrix of $t_{n}$.

Experiment Results. Table 4 shows the results of the emotion prediction experiment. The baselines are observed to performed poorly, achieving only $0.035 \mathrm{~F} 1$ scores for both Multinomial NB and SVC. ET-HMM is observed to outperformed the baselines, achieving 0.213 for F1 score. We postulate that the ET-HMM model's superior performance could be attributed to its consideration sequential information.

\section{Discussion}

In this study, we analyzed emotion dynamics in Twitter during the COVID-19 pandemic, characterizing topics, emotions and their transitions. In our dataset, we observe a sharp increase in the number of tweets collected from the week of March 22 to March 29. During this week, at least eight states in the US have issued stay-at-home orders, and many other countries begin movement restrictions, which might cause the great outpouring of yearning expressed on Twitter as people adjust to staying and working at home. Another week of note is April 19 to May 3, where countries like Spain, Italy and Malaysia extend stay-at-home orders due to persistent rise in cases. We postulate the decrease in the number of tweets may be due to adjustments to new routines and people have leveraged digital technologies to overcome the yearning for family 
Table 3: Dominant topics per emotion transition

\begin{tabular}{|l|l|l|l|}
\hline $\begin{array}{l}\text { Erratic transitions } \\
\text { (short time period) }\end{array}$ & Disgust $\rightarrow$ Anger & Optimism $\rightarrow$ Joy & $\begin{array}{l}\text { Erratic transitions } \\
\text { (long time period) }\end{array}$ \\
\hline Topic 2 & Topic 7 & Topic 9 & Topic 7 \\
vaccine & trump & patient \\
testing & elections & doctor \\
hospital & healthcare worker & free \\
treatment & free & mask \\
drug & mask & Topic 1 & Topic 9 \\
\hline Topic 6 & Topic 9 & medium & doctor \\
death & patient & news & hospital \\
case & doctor & government & \\
total & hospital & fact & \\
rate & healthcare worker & & \\
& & & \\
\hline
\end{tabular}

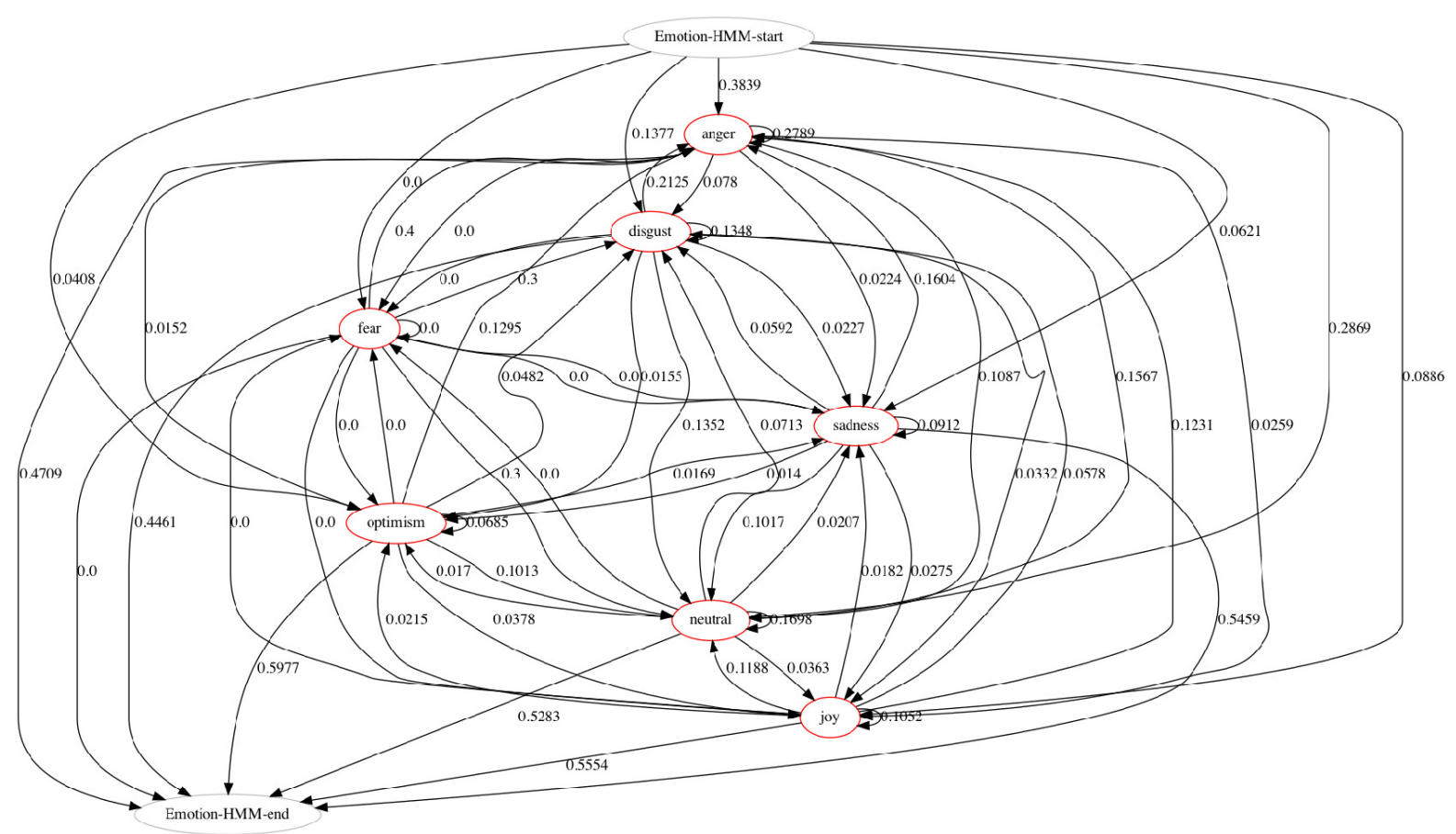

Figure 5: Hidden Markov Model of Emotions with Topics as Hidden States

Table 4: Emotion prediction experiment results.

\begin{tabular}{|l|l|l|l|}
\hline Model & Prec. & Rec. & Micro-F1 \\
\hline Multinomial NB & 0.031 & 0.048 & 0.035 \\
\hline SVC & 0.032 & 0.047 & 0.035 \\
\hline ET-HMM & 0.241 & 0.375 & 0.213 \\
\hline
\end{tabular}

and friends.

Topic Discovery. From initial filtering of tweets based on word related to yearning, we observe that Twitter users find hope and love, yet feel helpless and turn to religion. Many users miss their loved family and friends and activities like summer, school and recreational play. This is not unusual in a lockdown situation.
The topic analysis in the final corpus reflects themes that users are concerned about during these emotional times (Table 1 and Figure 2). Users reflect worry for the medical progress of vaccines, compassion for healthcare workers at the frontlines of the crisis and worry about their economic situation (i.e. businesses and jobs). This pandemic coincides with the 2020 US elections, which is discussed extensively. Another key topic of discussion is the lockdown situation and personal relationships, because people are unable to see their loved ones face-to-face.

Emotion Dynamics. With emotion dynamics, 
we observe that emotions are not strongly correlated with topics, where some topics are discussed more extensively when expressing certain emotions. The dominant topic distribution is different when considering singular emotions dynamics and longitudinal emotions dynamics.

Tweets exhibiting the emotion fear generally discuss topics 9 and 10 (concern for healthcare workers and business/ job reliefs). The topic of relationships dominates tweets with emotions of joy and sadness. These observations situate emotional states when talking about economic situation and loved ones, providing a preliminary characterization into the topics prevalent for each emotion.

Emotion Transitions. In a helpless pandemic situation, it is not uncommon for a person to express high amounts of disgust and anger, and oscillate between both states or remain in those states. Our results show that the (disgust $\rightarrow$ anger) transition and self-loops of (disgust $\rightarrow$ disgust) and (anger $\rightarrow$ anger) are observed significantly more than other emotion transition pair. Disgust-Anger falls into first category of Pluchik's colour wheel of emotions, which means they are most often felt and expressed together (Kołakowska et al., 2015). This emotion pair suggest contempt of the situation.

Our study of "mood swing" patterns indicate that emotion dynamic trails in Cluster 2 (disgust $\rightarrow$ anger) mostly tweet about the government's handling of the situation and social issues arising from lockdown like starvation and strike. Trails in Cluster 3 (optimism $\rightarrow$ joy) mostly tweet selfhelp and motivational tweets, while erratic trails typically present a twitter-log of the user's lives. It is interesting to note that many accounts have defined personas as inferred from their emotion dynamic trails, and a person's twitter-log reflects fluctuations of emotions, or "mood swings".

Predicting Emotion Dynamics. A random user is most likely to begin his emotion dynamic trail with an angry tweet, reflecting how people turn to social media to vent their frustration of movement control. With trails ending with all emotions except fear, we postulate that tweeting and the interaction from can diffuse anger and change users' emotions.

Our ET-HMM framework correctly classified $21 \%$ of the emotions of the test tweets respectively, outperforming baseline classifiers. A possible reason for ET-HMM superior performance could be the model's consideration of sequential information. ET-HMM is able to learn the sequential in- formation from the transition of topic and emotion in users' tweets. Nevertheless, the current prediction task does not account for advance temporal properties between tweets, i.e. accounting where a prolonged time between tweets may affect the emotions expressed differently. Future research could utilize the sequential alignment of emotion trails and integrating additional factors like temporal or geographical information that may affect the emotion class.

Limitations. As with any study, there are several limitations of this work. In particular, the corpus presents a highly skewed data of users. $45 \%$ of users have less than 2 tweets, while less than $1 \%$ of users have over 5000 tweets. The corpus also presents a skewed representation of emotions, which makes characterising emotions difficult: disgust and anger tweets comprise of $67 \%$, while only $0.0004 \%$ of tweets exhibit fear. Nonetheless, there are promising directions stemming from this work, such as harnessing time-series and user network information in emotion profiling and emotion dynamics prediction.

\section{Bye, Iill miss you babe: Conclusion}

In this paper, we characterised Twitter users' emotion dynamics during the COVID-19 pandemic in March 2020 by performing longitudinal user studies profiling topics discussed and the emotions exhibited. During this time period, disgust and anger are the most commonly expressed emotions, and that user emotion transitions tend to exhibit four main "mood swing" profiles. We construct an Emotion Topic Hidden Markov Model to reflect the importance of tweet content on emotional state, presenting a model that outperforms baselines. We hope our work sheds light on user emotions during a pandemic, and will inspire future directions in monitoring emotional states during isolation events, providing a means to identify high-risk individuals to offer emotional support through trying times.

For future work, we will consider tweet geographical location information in our analysis. For example, countries have different developments of the COVID-19 pandemic, and the varying health situation may elicit different emotions of Twitter users living in the country. Geolocation analysis may explain the changes in the users' emotional states at certain locations at certain times. We will also consider modeling emojis and other multimedia content in our topic and emotion analysis. 


\section{References}

Md Rabiul Awal, Rui Cao, Sandra Mitrovic, and Roy Ka-Wei Lee. 2020. On analyzing antisocial behaviors amid covid-19 pandemic. arXiv preprint arXiv:2007.10712.

Haji Binali, Chen Wu, and Vidyasagar Potdar. 2010. Computational approaches for emotion detection in text. In 4th IEEE International Conference on Digital Ecosystems and Technologies, pages 172-177. IEEE.

Kathleen M. Carley. 2014. ORA: A Toolkit for Dynamic Network Analysis and Visualization, pages 1219-1228. Springer New York, New York, NY.

Niko Colneriê and Janez Demsar. 2018. Emotion recognition on twitter: Comparative study and training a unison model. IEEE transactions on affective computing.

Aparna Gullapalli and Kathleen M. Carley. 2013. Extracting ordinal temporal trail clusters in networks using symbolic time-series analysis. Social Network Analysis and Mining, 3(4):1179-1194.

Raj Kumar Gupta, Ajay Vishwanath, and Yinping Yang 2020. Covid-19 twitter dataset with latent topics, sentiments and emotions attributes.

Vinay Kumar Jain, Shishir Kumar, and Steven Lawrence Fernandes. 2017. Extraction of emotions from multilingual text using intelligent text processing and computational linguistics. Journal of computational science, 21:316-326.

Nickolas M Jones, Sean P Wojcik, Josiah Sweeting, and Roxane Cohen Silver. 2016. Tweeting negative emotion: An investigation of twitter data in the aftermath of violence on college campuses. Psychological methods, 21(4):526.

Edward Chao-Chun Kao, Chun-Chieh Liu, Ting-Hao Yang, Chang-Tai Hsieh, and Von-Wun Soo. 2009. Towards text-based emotion detection a survey and possible improvements. In 2009 International Conference on Information Management and Engineering, pages 70-74. IEEE.

Agata Kołakowska, Agnieszka Landowska, Mariusz Szwoch, Wioleta Szwoch, and Michał R Wróbel. 2015. Modeling emotions for affect-aware applications. Information Systems Development and Applications, pages 55-69.

Puja Mehta, Daniel F McAuley, Michael Brown, Emilie Sanchez, Rachel S Tattersall, and Jessica J Manson. 2020. Covid-19: consider cytokine storm syndromes and immunosuppression. The Lancet, 395(10229):1033-1034.

David Mimno and Maintainer David Mimno. 2013. Package 'mallet'. A wrapper around the Java machine learning tool MALLET.
Saif M. Mohammad, Felipe Bravo-Marquez, Mohammad Salameh, and Svetlana Kiritchenko. 2018. Semeval-2018 Task 1: Affect in tweets. In Proceedings of International Workshop on Semantic Evaluation (SemEval-2018), New Orleans, LA, USA.

Rajdeep Mukherjee, Sriyash Poddar, Atharva Naik, and Soham Dasgupta. 2020. How have we reacted to the covid-19 pandemic? analyzing changing indian emotions through the lens of twitter.

Debashis Naskar, Eva Onaindia, Miguel Rebollo, and Subhashis Das. 2019. Modelling emotion dynamics on twitter via hidden markov model. In Proceedings of the 21st International Conference on Information Integration and Web-based Applications \& Services, pages 245-249.

Debashis Naskar, Sanasam Ranbir Singh, Durgesh Kumar, Sukumar Nandi, and Eva Onaindia de la Rivaherrera. 2020. Emotion dynamics of public opinions on twitter. ACM Transactions on Information Systems (TOIS), 38(2):1-24.

Jeffrey Pennington, Richard Socher, and Christopher D. Manning. 2014. Glove: Global vectors for word representation. In Empirical Methods in Natural Language Processing (EMNLP), pages 1532-1543.

Lawrence R Rabiner. 1989. A tutorial on hidden markov models and selected applications in speech recognition. Proceedings of the IEEE, 77(2):257286.

Kashfia Sailunaz and Reda Alhajj. 2019. Emotion and sentiment analysis from twitter text. Journal of Computational Science, 36:101003.

Kashfia Sailunaz, Manmeet Dhaliwal, Jon Rokne, and Reda Alhajj. 2018. Emotion detection from text and speech: a survey. Social Network Analysis and Mining, 8(1):28.

Jacob Schreiber. 2018. pomegranate: Fast and flexible probabilistic modeling in python. Journal of $\mathrm{Ma}$ chine Learning Research, 18(164):1-6.

Elizabeth M Seabrook, Margaret L Kern, Ben D Fulcher, and Nikki S Rickard. 2018. Predicting depression from language-based emotion dynamics: longitudinal analysis of facebook and twitter status updates. Journal of medical Internet research, 20(5):e168.

G Subramaniam, $\mathrm{R}$ Aswini, $\mathrm{M}$ Ranjitha, and Praveen Kumar Rajendran. 2017. Survey on user emotion analysis using twitter data. In 2017 International Conference on Energy, Communication, Data Analytics and Soft Computing (ICECDS), pages 998-1001. IEEE.

Wei Wang, Ivan Hernandez, Daniel A Newman, Jibo He, and Jiang Bian. 2016. Twitter analysis: Studying us weekly trends in work stress and emotion. $A p$ plied Psychology, 65(2):355-378. 